\title{
Separating FECG from MECG and Analyzing Fetal Heart Rate
}

\author{
Farhad Ahmad Mir'1, and Monika Mehra² \\ ${ }^{1}$ M.Tech. Student, Department of Electronics and Communication Engineering, RIMT University, Punjab, India \\ ${ }^{2}$ Associate Professor, Department of Electronics and Communication Engineering, RIMT University, Punjab, India \\ Correspondence should be addressed to Farhad Ahmad Mir; mirfarhadnabi@yahoo.com
}

Copyright (C) 2022 Farhad Ahmad Mir et al. This is an open-access article distributed under the Creative Commons Attribution License, which permits unrestricted use, distribution, and reproduction in any medium, provided the original work is properly cited.

\begin{abstract}
Figuring out fetal coronary heart can be essential for monitoring the situation of a fetus. While an ECG signal is recorded by way of putting electrodes on the maternal stomach, the fetal ECG is regularly masked by using the maternal ECG sign and noise. Via using an LMS adaptive filter to extract the fetal ECG signal, after which the use of the Pan \& Tompkins algorithm to hit upon the QRS complexes, the fetal heart may be determined with very low errors.
\end{abstract}

KEYWORDS: FECG. MECG, Heart rate, fetal coronary heart

\section{INTRODUCTION}

It is essential for tracking matters and nicely-being of a fetus, because it can aid in detecting arrhythmias or different such troubles. One among the foremost important portions of knowledge that this provides is whether or not the infant is receiving sufficient oxygen. To find the fetal coronary heart charge, a Doppler ultrasound is usually used at some stage within the pregnancy. This approach consists, because the decision indicates, of using ultrasound waves that are meditated by means of the fetal pulse rate, altering the frequency of the waves. The Doppler transforms this into a legitimate, that the heart rate is also heard and should be displayed on a display. However, this system is not appropriate for long Electrocardiograms, or ECGs, are wont to report electric pastime of the center. Signals containing maternal and indicators is also obtained with the help of setting electrodes on the maternal stomach. But, there are sure challenges while attempting to extract the fetal ECG sign from a sign containing the maternal and fetal ECG signals additionally to noise. The amplitude of the QRS of the fetal signal is enough smaller (order of microvolts) as compared to the maternal signal (order of millivolts) [2]. This additionally manner that irrespective of where electrodes are positioned at the body, the mom's heartbeat will still generate noise that masks the sign of the fetus.

To overcome those challenges and within the end decide the fetal heart price, exceptional strategies were implemented. To realize the fetal ECG signal, a crosscorrelation feature has been used, an averaged maternal ECG waveform became derived, after which a template sign of the maternal ECG turned into created the employment of the averaged ECG waveform. To retrieve the averaged fetal ECG, the maternal ECG was subtracted from the unique signal [3]. To de-noise the signal, the wavelet transform has been used. The wavelet analysis is simply like Fourier evaluation; however, at the identical time as $f(t)$ breaks the symptoms into a series of sine waves at distinct frequencies, the wavelet remodel breaks the sign into "wavelets" and uses internal products to degree similarities among the wavelets and signal [4]. Other strategies which are applied include impartial element evaluation [5] and also the pan and Tompkins set of rules [6] which contain the usage of diverse filters, amplification strategies, and sign looking algorithms to extract the fetal ECG signal. Our proposed method, shown in fig. 1, are to use a Least Mean Square (LMS) adaptive filter to get just the fetal ECG signal, then the newly obtained signal are

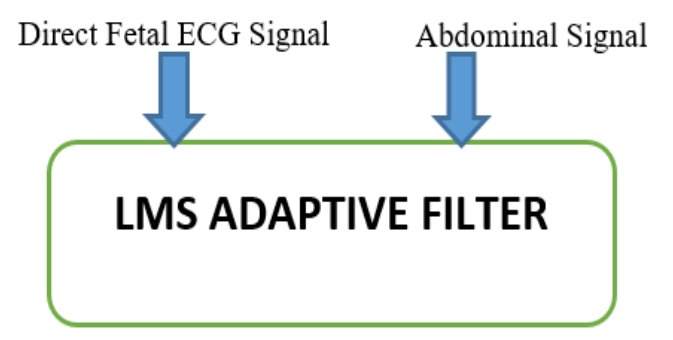

Filtered

Abdominal

Signal

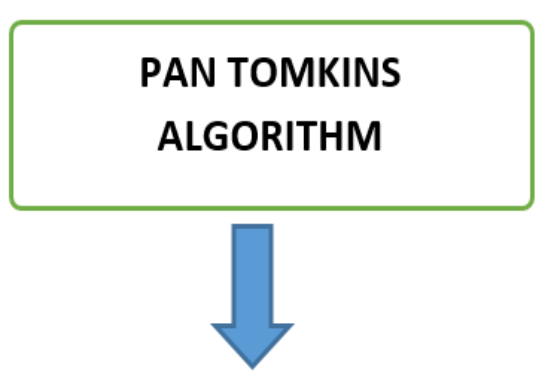

Filtered FECG

Figure 1. Proposed Method Block Diagram

processed with Pan and Tompkins algorithm to search out the QRS complexes and thus obtain the heart rate. 


\section{METHOD}

The proposed method was developed and tested using the Abdominal and Direct Fetal Electrocardiogram Database from PhysioNet. The database contains multichannel fetal ECG recordings from 5 different women. Each woman was aborning and between 38 and 41 weeks of gestation when the recordings were obtained. Each recording consists of 4 signals acquired from the maternal abdomen additionally to an immediate fetal ECG recorded from the fetal head. The four abdominal electrodes were placed round the navel while a reference electrode was placed above the pubic symphysis. A reference electrode was also placed on the left leg to ground the signal. The bandwidth of the signals was $1-150 \mathrm{~Hz}$, the rate was $1 \mathrm{kHz}$, and also the resolution is 16 bits [7].

\section{A. Least Mean Square Adaptive Filter}

The main idea behind adaptive filtering is that a variable filter (shown as wn in fig 2.) is adjusted until the difference between the variable filter output and also the desired signal is as small as possible. Fig. 2 shows the adaptive filter diagram. An abdominal signal, $\mathrm{x}(\mathrm{n})$, is suffered the variable filter, which alters $\mathrm{x}(\mathrm{n})$ in line with $\mathrm{w}(\mathrm{n})$, a vector composed of weights that controls the filter [8]. The output of the altered signal, represented by y (n), is then compared to $d(n)$, the required output. The difference between y (n) and d (n) is e (n), or the mean-square error. Next, the error signal and $\mathrm{x}(\mathrm{n})$ withstand the adaptive algorithm, which updates the filter coefficients to aim to further reduce the error [9].To update the filter coefficients (wn), the adaptive algorithm depends on the parameter $\mu$. this can be the step size of the adaptive filter. It affects the convergence speed (how quickly the error signal is minimized) and also the steady state error (because the adaptive filter isn't perfect,

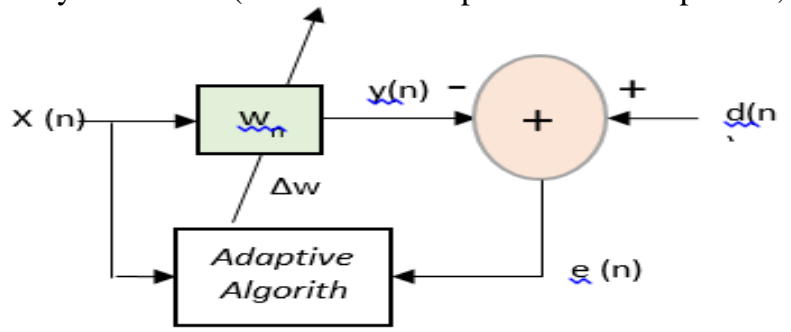

Figure 2. LMS Adaptive Filter Block Diagram

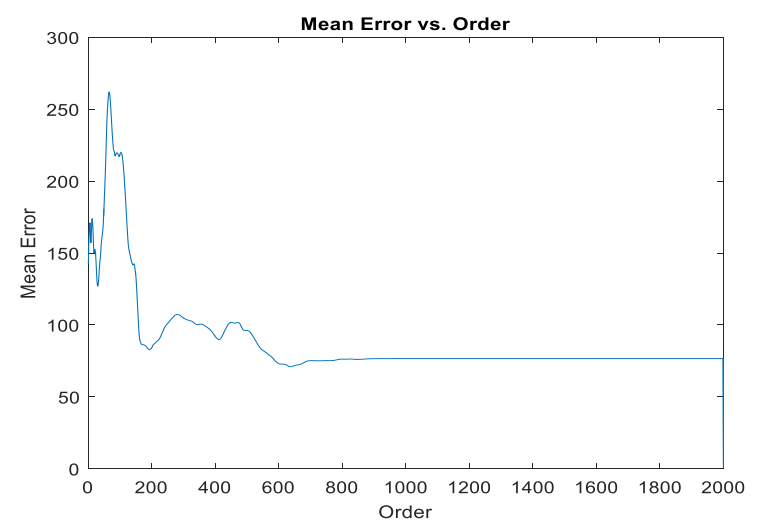

Figure 3. Mean Error vs. Order the error might not converge exactly to zero). A little step size corresponds to a little steady state error. However, it decreases the convergence speed [9]. When extracting the fetal ECG signal from the abdominal signal, the step sizes that were tested ranged from $0.40-0.85$. From this range, the step size that provided the smallest amount percent error once fetal rate was calculated was chosen. One other parameter that affects the adaptive filter is the order, or length, of the weight vector.

Increasing the order should lead to less noise, but it can even make the fetal heartbeat magnitude tougher to detect [10]. Additionally, the larger the order, the longer the time interval of the filter. When determining the order to use, the mean error was compared to the order, and as exemplified by fig 3 . When the order was greater than approximately 800 , the mean error was constant. The order that was used was 1000 .

\section{B. Pan \& Tompkins Algorithm}

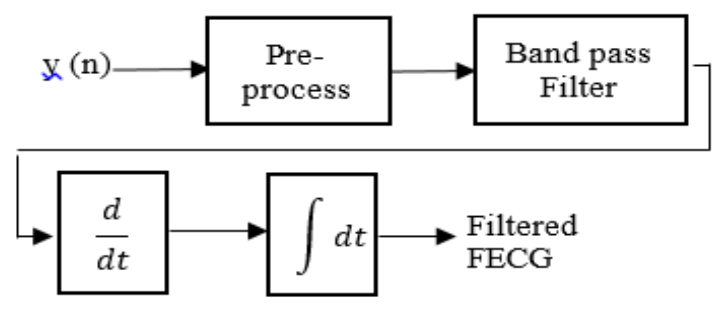

Figure 4. Pan and Tompkins Algorithm Block Diagram

Once the fetal ECG signal has been extracted from the abdominal signal, the Pan and Tompkins algorithm is employed to locate the $\mathrm{R}$ waves (the diagram can are often seen in fig. 4.).

The algorithm first pre-processes the signal to confirm that the frequency is $200 \mathrm{~Hz}$. Then a band pass filter with a passband of $5-15 \mathrm{~Hz}$ is employed to filter noise. Although the signal has already been filtered by the adaptive filter, this extra filtration may aid within the removal of any lingering noise within this frequency range [1], [2].

Next, a differentiator is employed. With differentiation, the signal will be smoothed and also the signal to noise ratio are often increased. Additionally, this will aid in peak detection. By determining the points where the signal crosses the $\mathrm{x}$-axis (zero crossings), and by determining if the slope is bigger than a established slope threshold (again to assist reduce the effect of noise), the possible peaks of the signal are often located [8]. The new signal is then squared to get positive values and, more importantly, to stress the larger frequencies [2].

After differentiation, and integrator is required. The Pan and Tompkins algorithm uses a moving-window integration to realize more information on the waveform [2]. With the placement of the $\mathrm{R}$ waves known, vital sign (beats/minute) may be calculated as $1000 *(60) /(\mathrm{RR}$ interval (milliseconds)). the tactic is applied to every of the four signals obtained from the four electrodes, and therefore the calculated heart rates are often compared to the direct fetal ECG signal that's recorded from the electrode placed on the fetal scalp. To work out the accuracy of the tactic, percent error is calculated. 


\section{RESULTS}

Table 1: Calculated fetal heart rates for Patient 07

\begin{tabular}{|l|r|l|r|}
\hline Channel & Fetal Heart Rate (bpm) & mu & Percent Error \\
\hline Direct & 125.2091 & & \\
\hline $\mathrm{Ab} \mathrm{1}$ & 126.6114 & 0.58 & 1.12 \\
\hline $\mathrm{Ab} \mathrm{2}$ & 126.6127 & 0.83 & 1.12 \\
\hline $\mathrm{Ab} \mathrm{3}$ & 124.4120 & 0.41 & 0.96 \\
\hline $\mathrm{Ab} \mathrm{4}$ & 125.8101 & 0.76 & 0.48 \\
\hline
\end{tabular}

Table I shows the fetal rate calculated using the direct signal, the fetal pulse calculated using each abdominal signal, the step size utilized to obtain each vital sign, and also the percent error between the reference pulse rate and therefore the calculated pulse rate for one patient. Figs. 5, 6 , and seven show an abdominal signal, the required output, and also the sign, respectively, for the identical patient. The signalling shown in fig. 7 closely resembles the required output shown in fig. 6. The effectiveness of the filtering method can even be seen by the low percent errors shown within the table. For every abdominal. Calculated fetal heart rates for Patient 07. Signal tested for this patient, the percent error was below $2 \%$. Overall, nearly all the calculated fetal heart rates had a percent error under $5 \%$. It should be noted that the step sizes did vary noticeably, and calculating the guts rate for every abdominal signal required a extended time interval.

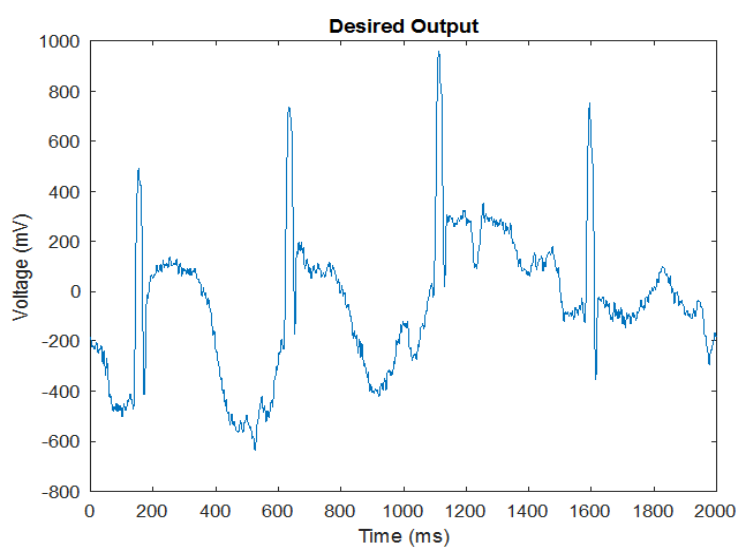

Figure 5. Abdominal signal obtained from Patient 07

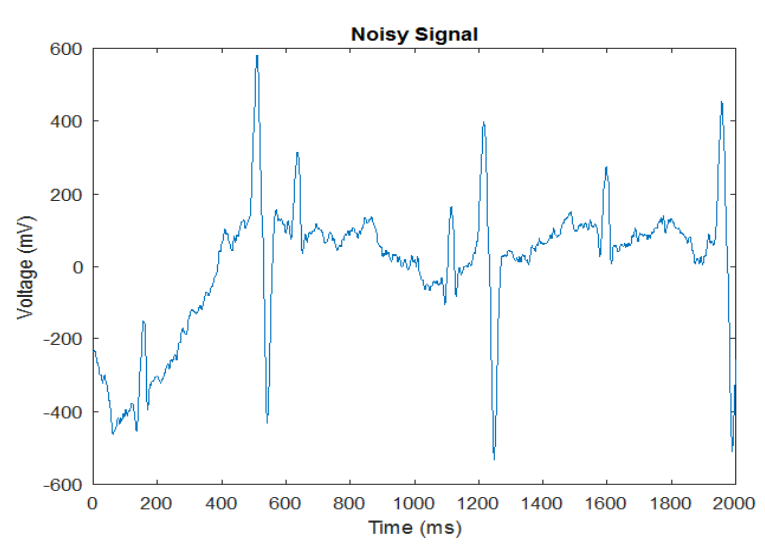

Figure 6. Signal obtained from the direct fetal scalp electrode from Patient 07

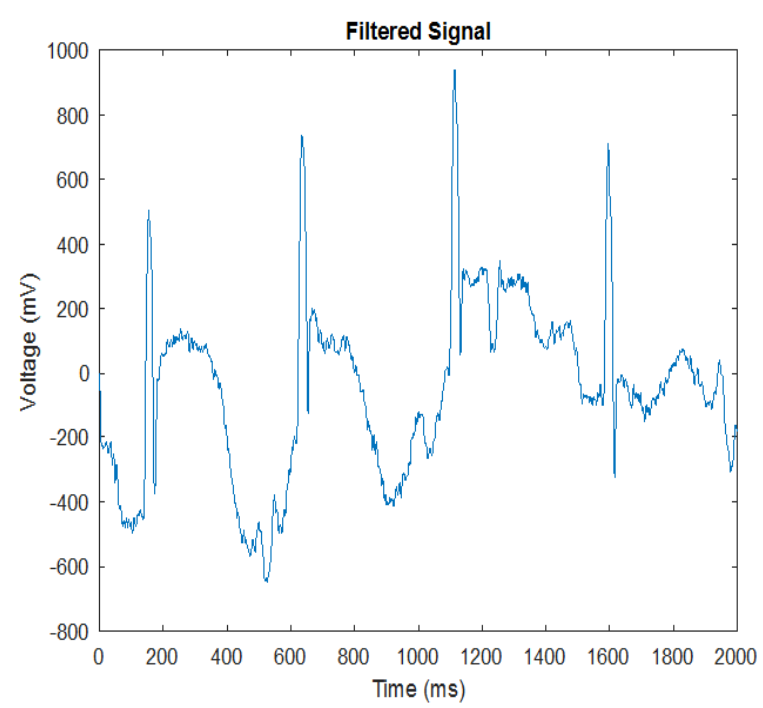

Figure 7. Output signal after using the LMS adaptive filter for Patient 07

Table 2: Calculated fetal heart rate using the average abdominal signal for Patient 07

\begin{tabular}{|l|r|r|}
\hline Channel & Fetal Heart Rate $(\mathrm{mu}=0.83)$ & Percent Error \\
\hline Direct & 125.2091 & \\
\hline Abs & 126.8118 & 1.28 \\
\hline
\end{tabular}

Table 3: Calculated fetal heart rate using the average abdominal signal for Patient 08

\begin{tabular}{|lr|r|}
\hline Channel & Fetal Heart Rate $(\mathrm{mu}=0.54)$ & Percent Error \\
\hline Direct & 130.1175 & \\
\hline Abs & 131.1193 & 0.77 \\
\hline
\end{tabular}

\section{USING AVERAGED ABDOMINAL SIGNAL}

To decrease the time required to calculate the fetal vital sign, the four abdominal signals were averaged. As a result, only 1 step size needed to be found. Tables II and III show the results of two patients. Overall, the percent error for one patient was $5.31 \%$, but the opposite four patients had percent errors below $5 \%$.

\section{CONCLUSION}

It has been shown that the LMS adaptive filter, together with the Pan and Tompkins algorithm, is effective in determining fetal pulse rate. However, a limitation of the strategy explored during this proposal is that an on the spot fetal scalp signal is required for the required output of the adaptive filter. The optimal method for determining fetal vital sign would involve a noninvasive approach, as this could afford greater comfort for the mother, greater ease when attaching the electrodes, and also the method may well be used before labor. A possible modification to the method would be to use the maternal ECG, obtained from electrodes placed on the mother's chest cavity, because the desired output. If the input remained the same - - the abdominal signal that contains the maternal ECG, fetal ECG, and noise- then the error signal of the LMS adaptive 
filter would contain the fetal heartbeat. Using the Noninvasive Fetal ECG Database on PhysioNet, this modification was tested. The resulting fetal heart rates were, for the foremost part, within the proper range of 120$160 \mathrm{bpm}$. However, because there was no direct fetal scalp lead, the accuracy of those results cannot be determined. As such, this modification requires further exploration.

\section{REFERENCES}

[1] M. Ghodsi, H. Hassani, and S. Sanei, "Extracting fetal heart signal from noisy maternal ECG by multivariate singular spectrum analysis," Statistics and Its Interface, vol. 3, pp. 399-411. 2010.

[2] N. Marchon and G. Naik, "QRS detector for maternal abdominal ECG," 2016 International Conference on Signal and Information Processing (IConSIP), Vishnupuri, 2016, pp.

[3] S. Abboud, A. Alaluf, S. Einav, D. Sadeh, "Real-Time Abdominal Fetal ECG Recording Using a Hardware Correlator,"

[4] V. Vigneron, A. Paraschiv-Ionescu, A. Azancot, O. Sibony and C. Jutten, "Fetal electrocardiogram extraction based on non-stationary ICA and wavelet denoising," Seventh International Symposium on Signal Processing and Its Applications, 2003. Proceedings., 2003.

[5] J. Szalai and F. E. Mozes, "Determining fetal heart rate using independent component analysis," 2014 IEEE 10th International Conference on Intelligent Computer Communication and Processing (ICCP).

[6] J. Pan and W. J. Tompkins, "A Real-Time QRS Detection Algorithm," in IEEE Transactions on Biomedical Engineering, vol. BME-32, no. 3, pp. 230-236, March 1985.

[7] B. Paul and P. Mythili, "ECG noise removal using GA tuned sign-data least mean square algorithm," 2012 IEEE International Conference on Advanced Communication Control and Computing Technologies (ICACCCT), Ramanathapuram, 2012, pp. 100-103. doi: 10.1109/ICACCCT.2012.6320750

[8] Ni.com. (2017). Least Mean Square (LMS) Adaptive Filter -National Instruments. [online] Available at: http://www.ni.com/example/31220/en/ [Accessed 16 Dec. 2017].

[9] M. Wasimuddin and N. Gupta, "Design and implementation of Least Mean Square adaptive filter on fetal electrocardiography," Proceedings of the 2014 Zone 1 Conference of the American Society for Engineering Education, Bridgeport, CT, 2014, pp. 1-5. doi: 10.1109/ASEEZone1.2014.6820650 